\title{
Magnetic field line random walk in two-dimensional dynamical turbulence
}

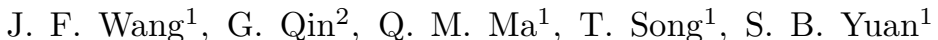 \\ ${ }^{1}$ Research Department of Biomedical Engineering, \\ Institute of Electrical Engineering, Chinese Academy of Science, \\ Beijing 100190, China; wangjunfang@mail.iee.ac.cn \\ ${ }^{2}$ School of Science, Harbin Institute of Technology, Shenzhen 518055, China; qingang@hit.edu.cn
}

(Dated: July 3, 2017)

The field line random walk (FLRW) of magnetic turbulence is one of the important topics in plasma physics and astrophysics. In this article by using the field line tracing method mean square displacements (MSD) of FLRW is calculated in all possible length scales for pure two-dimensional turbulence with damping dynamical model. We demonstrate that in order to describe FLRW with damping dynamical model a new dimensionless quantity $R$ is needed to be introduced. In different length scales dimensionless MSD shows different relationship with the dimensionless quantity $R$. Although temporal effect impacts MSD of FLRW and even changes regimes of FLRW, it dose not affect the relationship between the dimensionless MSD and dimensionless quantity $R$ in all possible length scales.

PACS numbers: 47.27.tb, 96.50.Ci, 96.50.Bh 


\section{INTRODUCTION}

Field line random walk (FLRW) or field line wandering of magnetic turbulence is one of the major problems in the study of the galactic confinement of cosmic rays (see, e.g., Jokipii \& Parker 1969), particle acceleration at perpendicular shocks (see, e.g., Giacalone \& Jokipii 1996), heat conduction by electrons (see, e.g., Chandran \& Cowley 1998; Lazarian 2006), and many other astrophysical applications. It has been demonstrated in several articles that FLRW directly affects the transport of charged particles (see, e.g., Skilling et al 1974; Giacalone \& Jokipii 1999; Kóta \& Jokipii 2000; Matthaeus et al 2003; Shalchi \& Kourakis 2007a; Shalchi \& Kourakis 2007b; Shalchi et al 2007). So the knowledge of field line wandering is also important for understanding the properties of energetic charged particles propagation through the interplanetary or interstellar system and ion diffusion in fusion devices (see, e.g., Kóta \& Jokipii 2000; Schlickeiser 2002; Matthaeus et al 2003; Webb et al 2006; Qin 2007; Shalchi et al 2007; Shalchi \& Kourakis 2007c; Tautz et al 2008; Shalchi et al 2009; Weinhorst \& Shalchi 2010; Webb et al 2009; Buffie \& Shalchi 2012; Qin \& Zhang 2014).

According to observations the total magnetic field of the magnetized plasma is usually considered as the superposition of a mean magnetic field $B_{0}$ and a turbulent component $\delta \vec{B}$. Such magnetic field configuration can be found in the solar system, the Galactic space and fusion devices, e.g., Tokamaks. Since the middle of the last century various analytical theories have been developed to describe field line wandering in such magnetic field configuration. The first one is the quasilinear theory which can be understood as a first-order perturbation theory (see, Jokipii 1966). However, the quasilinear theory only works in pure slab (one-dimensional) magnetic turbulence for parallel transport with flat spectrum and for perpendicular diffusion if pitch-angle scattering is suppressed and if the Kubo number is small (Shalchi 2015). For two-dimensional, quasi-3D (three-dimensional) and 3D magnetic turbulence the quasilinear theory is problematic to compute FLRW and nonlinear description is essential (Shalchi 2009). So Matthaeous et al (1995) developed a nonlinear analytical theory for FLRW based on the diffusion assumption. But the superdiffusive regime of field line wandering was soon found by computer simulations (see, e.g., Zimbardo et al 1995; Pommois et al 1999). Therefore, the diffusion theory of Matthaeous et al (1995) had to be extended to describe nondiffusion regime (see, Shalchi \& Kourakis 2007a). Whereafter, some articles demonstrated that the energy range index of the turbulence spectrum determines whether random walk on magnetic field lines is diffusive or not (see, e.g., Shalchi \& Kourakis 2007b, Shalchi \& Weinhost 2009), which was confirmed by Shalchi \& Qin (2010). Furthermore, it was found by analytical investigations that the spectral anisotropy is another important factor affecting field line wandering (see Weinhorst \& Shalchi 2010). But it is not clear that the most realistic regimes of field line wandering is superdiffusive or diffusive. With regard to the confusion about the regimes of field line wandering, it was clarified by the in-depth study of Shalchi (2011) that random walk on magnetic field lines complies with different transport regimes for different length scales.

The papers listed above only explored FLRW of magnetostatic turbulence. But the real magnetic turbulence should be time dependent, and it has been found that dynamical turbulence effects have an important influence on FLRW and transport of energetic charged particle (see Shalchi 2009). For slab turbulence with plasma wave model Shalchi et al (2007) obtained a classic diffusive result for random walk on magnetic field lines. Later, with regard to damping model and random sweeping model Shalchi (2010a) found that dynamical models are less restrictive concerning the allowed energy range index than magnetostatic case for transport regime of field line wandering. And for pure two-dimensional turbulence with damping dynamical model and nonlinear anisotropic dynamical turbulence model Guest \& Shalchi (2012) found that both energy range index $q$ and the choice of the dynamical models have an strong impact on the field line wandering. The articles mentioned above only explored the features of FLRW in large spatial scale. Shalchi (2011) investigated the properties of FLRW for pure two-dimensional magnetostatic turbulence in all possible length scales, i.e., inertial range, energy range and even the range larger than the box scale. But the influence of temporal effect on field line wandering in all possible spatial length scales is an open topic, and the implicit dimensionless quantity controlling FLRW of dynamical turbulence is also an unresolved issue. The purpose of this article is to explore the these problems. For mathematical tractability in this paper we only investigate the impact of the simple damping model with constant temporal factor on FLRW of pure two-dimensional turbulence.

The organization of the article is as follows. In Section II we show the pure two-dimensional magnetic turbulence mode. In Section III we describe the theory of FLRW developed by Shalchi \& Kourakis (2007a). In Section IV we extend the basic formulas of FLRW in all possible spatial length scales for pure two-dimensional magnetostatic turbulence (Shalchi 2011) to dynamical turbulence. In Section V, Section VI and Section VII we derive the specific analytical formulas of mean square displacement of field line wandering in different length scales. Section VIII shows summary and conclusion. 


\section{PURE TWO-DIMENSIONAL TURBULENCE MODEL}

In this article we explore field line wandering of pure two-dimensional dynamical turbulence. And the turbulent magnetic field component $\delta \vec{B}(\vec{x})$ is oriented perpendicular with respect to the mean magnetic field, i.e., $\delta B_{z}=0$. The tensor of the 2D (two-dimensional) magnetic turbulence has the following form (see Shalchi 2009)

$$
P_{l m}^{2 D}(\vec{k})=g^{2 D}\left(k_{\perp}\right) \frac{\delta\left(k_{\|}\right)}{k_{\perp}}\left[\delta_{l m}-\frac{k_{l} k_{m}}{k^{2}}\right], \quad l, m=x, y
$$

where $\delta_{l m}$ is the Kronecker delta and $\delta\left(k_{\|}\right)$is the Dirac delta. And $g^{2 D}\left(k_{\perp}\right)$ is the spectrum of the two-dimensional modes adopted in Shalchi (2011)

$$
g^{2 D}\left(k_{\perp}\right)=\frac{D(s, q)}{2 \pi} l_{2 D} \delta B_{2 D}^{2} \begin{cases}0, & k_{\perp}<L_{2 D}^{-1} \\ \left(k_{\perp} l_{2 D}\right)^{q}, & L_{2 D}^{-1}<k_{\perp}<l_{2 D}^{-1} \\ \left(k_{\perp} l_{2 D}\right)^{-s}, & l_{2 D}^{-1}<k_{\perp} .\end{cases}
$$

Here $l_{2 D}$ denotes the turnover or bendover scale, $L_{2 D}$ stands for box scale which denotes the largest scale of the stochastic magnetic field system, $\delta B_{2 D}$ is the field strength of the two-dimensional modes, $s$ is the inertial range spectral index, and $q$ is the energy range spectral index. The region between box scale and turnover scale is usually called energy range, and it is inertial range for $k_{\perp}>l_{2 D}^{-1}$. The normalization function is given by

$$
D(s, q)=\frac{(q+1)(s-1)}{s+q} .
$$

Here Eq. (2) is correctly normalized for $q>-1$ and $s>1$. In addition, we assume $l_{2 D} \ll L_{2 D}$ throughout this article.

\section{THEORY OF MAGNETIC FIELD LINE RANDOM WALK}

To study field line wandering of magnetic turbulence we have to use the method of statistical physics. And the mean square displacement (MSD) $\left\langle(\Delta x(z))^{2}\right\rangle$ of magnetic field line is the most frequently used physical quantity to describe the properties of turbulent magnetic field, where $\Delta x(z)=x(z)-x(0)$ is the cross-field distance and $\langle\cdots\rangle$ is ensemble average operator. In the most previous investigations, a power law $\left\langle(\Delta x)^{2}\right\rangle=\alpha|z|^{\beta}$ with positive constants $\alpha$ and $\beta$ is usually used to distinguish between different transport regimes according to different $\beta$, namely, $0<\beta<1$ for subdiffusion, $\beta=1$ for diffusion, $1<\beta<2$ for superdiffusion, and $\beta=2$ for ballistic process, where $|z|$ is the absolute value of distance along the mean magnetic field (see, e.g., Shalchi 2009). In what follows, we introduce the well-known theory of FLRW developed by Shalchi \& Kourakis (2007a).

The equation of the stochastic magnetic field line reads

$$
\frac{d x}{d z}=\frac{\delta B_{x}(\vec{x}, t)}{B_{0}}
$$

where $z$ and $x$ are the displacement parallel and perpendicular to the background magnetic field $\vec{B}_{0}$ respectively, and $\delta B_{x}(\vec{x}, t)$ denotes $x$-component of $\delta \vec{B}(\vec{x}, t)$.

From Eq. (4) the mean square displacement of magnetic field line can be written as

$$
\left\langle(\Delta x)^{2}\right\rangle=\frac{1}{B_{0}^{2}} \int_{0}^{z} d z^{\prime} \int_{0}^{z} d z^{\prime \prime}\left\langle\delta B_{x}\left(\vec{x}\left(z^{\prime}\right), t^{\prime}\right) \delta B_{x}\left(\vec{x}\left(z^{\prime \prime}\right), t^{\prime \prime}\right)\right\rangle .
$$

By operating Fourier transformation on the correlation tensor of magnetic turbulence the latter equation can be rewritten as

$$
\left\langle(\Delta x)^{2}\right\rangle=\frac{2}{B_{0}^{2}} \int d^{3} k P_{x x}(\vec{k}, t) \int_{0}^{z} d z^{\prime}\left(z-z^{\prime}\right)\left\langle e^{i \vec{k} \cdot \Delta \vec{x}(z)}\right\rangle,
$$

here spatial and temporal homogeneous assumptions and Corrsin independence hypothesis (see, Corrsin 1959) are used. Taking the second derivative of the latter equation over $z$ gives

$$
\frac{d^{2}\left\langle(\Delta x)^{2}\right\rangle}{d z^{2}}=\frac{2}{B_{0}^{2}} \int d^{3} k P_{x x}(\vec{k}, t)\left\langle e^{i \vec{k} \cdot \Delta \vec{x}(z)}\right\rangle .
$$


For mathematical tractability we assume all tensor components of turbulence obey same temporal behavior. Then we can obtain $P_{x x}(\vec{k}, t)=P_{x x}(\vec{k}, 0) \Gamma(\vec{k}, t)$ with the static tensor components $P_{x x}(\vec{k}, 0)$ and dynamical correction function $\Gamma(\vec{k}, t)$. Thus, we can get

$$
\frac{d^{2}\left\langle(\Delta x)^{2}\right\rangle}{d z^{2}}=\frac{2}{B_{0}^{2}} \int d^{3} k P_{x x}(\vec{k}, 0) \Gamma(\vec{k}, t)\left\langle e^{i \vec{k} \cdot \Delta \vec{x}(z)}\right\rangle .
$$

\section{FIELD LINE RANDOM WALK FOR PURE TWO-DIMENSIONAL TURBULENCE WITH DAMPING MODEL}

In this section we extend the basic formulas of FLRW in all possible spatial scales for pure two-dimensional magnetostatic turbulence (Shalchi 2011) to dynamical turbulence. For the sake of simplicity we only use the simple exponential decaying model for turbulent dynamical effect, i.e., the damping model

$$
\Gamma(\vec{k}, t)=e^{-\gamma t}
$$

where the parameter $\gamma$ is the characteristic temporal factor (see Bieber et al. 1994). For slab and two-dimensional turbulence temporal factor $\gamma$ is usually set as $\alpha v_{A} k$ with constant parameter $\alpha$, Alfvén wave speed $v_{A}$ and wave number $k$ (see Bieber et al. 1994; Shalchi 2010a; Guest \& Shalchi 2012). In this article for mathematical tractability we assume that the temporal factor $\gamma$ is a constant. We leave the case $\gamma=\alpha v_{A} k$ and other more complicated cases for future work.

By assuming the Gaussian distribution of the magnetic field line to evaluate the characteristic function $\left\langle e^{i \vec{k} \cdot \Delta \vec{x}(z)}\right\rangle$, Eq. (8) can be rewritten as

$$
\frac{d^{2}\left\langle(\Delta x)^{2}\right\rangle}{d z^{2}}=\frac{2}{B_{0}^{2}} \int d^{3} k P_{x x}(\vec{k}) e^{-\gamma t} e^{-\frac{1}{2}\left\langle(\Delta x)^{2}\right\rangle k_{\perp}^{2}} e^{i k_{\|} z}
$$

where we assume that magnetic turbulence is axisymmetric with respect to background magnetic field.

In this article we compute the mean square displacement of stochastic magnetic field line by employing the field line tracing method (see Shalchi 2010a), which assumes that an energetic charged particle or an aerocraft moves along magnetic field line with constant $z$-component speed $v=z / t$. By setting $\sigma=\left\langle(\Delta x)^{2}\right\rangle$ and substituting time $t$ with $z / v$ in Eq. (10), one can find

$$
\frac{d^{2} \sigma}{d z^{2}}=\frac{2}{B_{0}^{2}} \int d^{3} k P_{x x}(\vec{k}) e^{-\frac{\gamma}{v} z} e^{-\frac{1}{2} \sigma k_{\perp}^{2}} e^{i k_{\|} z}
$$

By using the tensor of the two-dimensional magnetic turbulence (see Eq. (11)), from Eq. (11) we can obtain the following equation

$$
\frac{d^{2} \sigma}{d z^{2}}=\frac{2 \pi}{B_{0}^{2}} \int_{0}^{\infty} d k_{\perp} g^{2 D}\left(k_{\perp}\right) e^{-\frac{\gamma}{v} z} e^{-\frac{1}{2} \sigma k_{\perp}^{2}}
$$

After multiplying Eq. (12) by $d \sigma / d z$, integrating the result by parts yields

$$
\begin{aligned}
\left(\frac{d \sigma}{d z}\right)^{2}= & \frac{8 \pi}{B_{0}^{2}} \int_{0}^{\infty} d k_{\perp} g^{2 D}\left(k_{\perp}\right) k_{\perp}^{-2}-\frac{8 \pi}{B_{0}^{2}} e^{-\frac{\gamma}{v} z} \int_{0}^{\infty} d k_{\perp} g^{2 D}\left(k_{\perp}\right) k_{\perp}^{-2} e^{-\frac{1}{2} \sigma k_{\perp}^{2}} \\
& -\frac{8 \pi}{B_{0}^{2}} \frac{\gamma}{v} \int_{0}^{z} d z^{\prime} e^{-\frac{\gamma}{v} z^{\prime}} \int_{0}^{\infty} d k_{\perp} g^{2 D}\left(k_{\perp}\right) k_{\perp}^{-2} e^{-\frac{1}{2} \sigma k_{\perp}^{2}},
\end{aligned}
$$

where the original conditions $\sigma(z=0)=0$ and $(d \sigma / d z)(z=0)=0$ are used. The first term on the right hand side of Eq. (13) is time-independent, but the second and third terms are affected by temporal effect. If setting $\gamma=0$ Eq. (13) is simplified as the magnetostatic turbulence result (see Eq. (16) in Shalchi 2011).

Substituting the formula of two-dimensional modes (see, Eq. (2)) into Eq. (13), and then using the transformation 
$y=l_{2 D} k_{\perp}$, finally one can obtain

$$
\begin{aligned}
\left(\frac{d \sigma}{d z}\right)^{2}= & 4 D(s, q) \frac{\delta B_{2 D}^{2}}{B_{0}^{2}} l_{2 D}^{2}\left(\frac{1-\xi^{q-1}}{q-1}+\frac{1}{s+1}\right) \\
& -2 D(s, q) \frac{\delta B_{2 D}^{2}}{B_{0}^{2}} l_{2 D}^{2} e^{-\frac{\gamma}{v} z} \\
& \times\left\{\rho^{1-q}\left[\Gamma\left(\frac{q-1}{2}, \rho^{2} \xi^{2}\right)-\Gamma\left(\frac{q-1}{2}, \rho^{2}\right)\right]+\rho^{s+1} \Gamma\left(-\frac{s+1}{2}, \rho^{2}\right)\right\} \\
& -2 D(s, q) \frac{\delta B_{2 D}^{2}}{B_{0}^{2}} l_{2 D}^{2} \frac{\gamma}{v} \\
& \times \int_{0}^{z} d z^{\prime} e^{-\frac{\gamma}{v} z^{\prime}}\left\{\rho^{1-q}\left[\Gamma\left(\frac{q-1}{2}, \rho^{2} \xi^{2}\right)-\Gamma\left(\frac{q-1}{2}, \rho^{2}\right)\right]+\rho^{s+1} \Gamma\left(-\frac{s+1}{2}, \rho^{2}\right)\right\},
\end{aligned}
$$

where the following formulas are used (see Gradshteyn \& Ryzhik 2007)

$$
\begin{aligned}
\int_{\xi}^{1} y^{q-2} d y & =\frac{1-\xi^{q-1}}{q-1}, \\
\int_{1}^{\infty} y^{-s-2} d y & =\frac{1}{s+1}, \\
\int_{\xi}^{1} y^{q-2} e^{-\rho^{2} y^{2}} d y & =\frac{1}{2} \rho^{1-q}\left[\Gamma\left(\frac{q-1}{2}, \rho^{2} \xi^{2}\right)-\Gamma\left(\frac{q-1}{2}, \rho^{2}\right)\right], \\
\int_{1}^{\infty} y^{-s-2} e^{-\rho^{2} y^{2}} d y & =\frac{1}{2} \rho^{s+1} \Gamma\left(-\frac{s+1}{2}, \rho^{2}\right) .
\end{aligned}
$$

Here $\Gamma(\nu, z)$ is the upper incomplete gamma function, the parameters $\xi=l_{2 D} / L_{2 D} \ll 1, \rho^{2}=\sigma /\left(2 l_{2 D}^{2}\right)$ and $\rho^{2} \xi^{2}=$ $\sigma /\left(2 L_{2 D}^{2}\right)$ are also used.

Upon two order differentiation on Eq. (14) with respect to $z$, we can get the ordinary differential equation of the mean square displacement which describes the properties of FLRW in all length scales

$$
\begin{aligned}
\frac{d^{2} \sigma}{d z^{2}}= & -\frac{D(s, q)}{2} \frac{\delta B_{2 D}^{2}}{B_{0}^{2}} e^{-\frac{\gamma}{v} z}\left\{\frac{1-q}{2}\left[\Gamma\left(\frac{q-1}{2}, \rho^{2} \xi^{2}\right)-\Gamma\left(\frac{q-1}{2}, \rho^{2}\right)\right]\left(\frac{2 l_{2 D}^{2}}{\rho^{2}}\right)^{\frac{1+q}{2}}\right. \\
& \left.+\left(e^{-\rho^{2}}-e^{-\rho^{2} \xi^{2}} \xi^{q-1}\right) \frac{2 l_{2 D}^{2}}{\sigma}+\frac{s+1}{2}\left(\frac{2 l_{2 D}^{2}}{\sigma}\right)^{\frac{1-s}{2}} \Gamma\left(-\frac{s+1}{2}, \rho^{2}\right)-e^{-\rho^{2}} \frac{2 l_{2 D}^{2}}{\sigma}\right\} .
\end{aligned}
$$

In the following sections, we consider the special cases in different length scales.

V. ANALYTICAL FORMULAS OF FIELD LINE WANDERING IN THE RANGE $\sigma \ll 2 l_{2 D}^{2} \ll 2 L_{2 D}^{2}$

In the range $\sigma \ll 2 l_{2 D}^{2} \ll 2 L_{2 D}^{2}$ we can see that the conditions $\rho^{2} \ll 1$ and $\rho^{2} \xi^{2} \ll 1$ need to be satisfied.

\section{A. The features of field line random walk in the range $\sigma \ll 2 l_{2 D}^{2} \ll 2 L_{2 D}^{2}$}

By using the conditions $\rho^{2} \ll 1$ and $\rho^{2} \xi^{2} \ll 1$ we can derive from Eq. (14)

$$
\begin{aligned}
\left(\frac{d \sigma}{d z}\right)^{2} \approx & 4 D(s, q) \frac{\delta B_{2 D}^{2}}{B_{0}^{2}} l_{2 D}^{2}\left(\frac{1-\xi^{q-1}}{q-1}+\frac{1}{s+1}\right)-4 D(s, q) \frac{\delta B_{2 D}^{2}}{B_{0}^{2}} l_{2 D}^{2} e^{-\frac{\gamma}{v} z} \times \\
& {\left[\frac{1-\xi^{q-1}}{q-1}+\rho^{2} \frac{\xi^{q+1}-1}{q+1}+\frac{\rho^{s+1}}{2} \Gamma\left(-\frac{s+1}{2}\right)+\frac{1}{s+1}+\frac{\rho^{2}}{1-s}\right] } \\
& -4 D(s, q) \frac{\delta B_{2 D}^{2}}{B_{0}^{2}} l_{2 D}^{2} \frac{\gamma}{v} \int_{0}^{z} d z^{\prime} e^{-\frac{\gamma}{v} z^{\prime}}\left[\frac{1-\xi^{q-1}}{q-1}+\rho^{2} \frac{\xi^{q+1}-1}{q+1}+\frac{\rho^{s+1}}{2} \Gamma\left(-\frac{s+1}{2}\right)\right. \\
& \left.+\frac{1}{s+1}+\frac{\rho^{2}}{1-s}\right]
\end{aligned}
$$


where the following formulas are used (see Abramowitz \& Stegun 1974)

$$
\begin{aligned}
& \Gamma(\nu, z \gg 1) \approx z^{\nu-1} e^{-z} \rightarrow 0, \\
& \Gamma(\nu, z \ll 1) \approx \Gamma(\nu)-\frac{z^{\nu}}{\nu}+\frac{z^{\nu+1}}{\nu+1} .
\end{aligned}
$$

By integrating by parts, Eq. (20) can be simplified as

$$
\left(\frac{d \sigma}{d z}\right)^{2} \approx 2 D(s, q) \frac{\delta B_{2 D}^{2}}{B_{0}^{2}}\left(\frac{1}{q+1}+\frac{1}{s-1}\right) \int_{0}^{z} d \sigma e^{-\frac{\gamma}{v} z^{\prime}}
$$

To differentiate Eq. (22) over z, one can obtain

$$
\frac{d^{2} \sigma}{d z^{2}} \approx \frac{\delta B_{2 D}^{2}}{B_{0}^{2}} e^{-\frac{\gamma}{v} z}
$$

By setting the original conditions as $(d \sigma / d z)(z=0)=0$ and $\sigma(z=0)=0$ as in above section, from Eq. (23) mean square displacement for the case $\sigma \ll 2 l_{2 D}^{2} \ll 2 L_{2 D}^{2}$ can be found

$$
\sigma \approx \frac{\delta B_{2 D}^{2}}{B_{0}^{2}} \frac{v}{\gamma}\left[z+\frac{v}{\gamma}\left(e^{-\frac{\gamma}{v} z}-1\right)\right]
$$

Note that FLRW in the range $\sigma \ll 2 l_{2 D}^{2} \ll 2 L_{2 D}^{2}$ is no longer the simple quadratic function of the parallel position $z$ (ballistic process) as in the magnetostatic case (see Shalchi 2011). In addition, we find that the energy range index $q$ and the inertial range index $s$ have no any influence on the features of field line wandering. In the following, we explore the properties of FLRW for some special cases in the range $\sigma \ll 2 l_{2 D}^{2} \ll 2 L_{2 D}^{2}$.

For the weak dynamical limit $\gamma \ll 1$ corresponding to the quasi-magnetostatic case, Eq. (24) is simplified as

$$
\sigma \approx \frac{1}{2} \frac{\delta B_{2 D}^{2}}{B_{0}^{2}} z^{2}
$$

The latter equation is the same as the magnetostatic result (see Eq. (26) in Shalchi 2011).

If temporal factor $\gamma$ is a nonzero value, from Eq. (24) we can get following equation in the interval $0<z \ll v / \gamma$

$$
\sigma \approx \frac{1}{2} \frac{\delta B_{2 D}^{2}}{B_{0}^{2}} z^{2}
$$

The latter equation is also identical with the magnetostatic case. So we can see that the temporal effect is negligible in the subrange $0<z \ll v / \gamma$ regardless of the strength of temporal effect. But if temporal effect is strong enough, the condition $v / \gamma \ll l_{2 D}$ can be satisfied, from Eq. (24) one can obtain the following formulas in the subrange $v / \gamma \ll z \ll l_{2 D}$

$$
\begin{aligned}
\kappa_{F L T} & \approx \frac{1}{2} \frac{\delta B_{2 D}^{2}}{B_{0}^{2}} \frac{v}{\gamma}, \\
\sigma & \approx \frac{\delta B_{2 D}^{2}}{B_{0}^{2}} \frac{v}{\gamma}\left(z-\frac{v}{\gamma}\right) .
\end{aligned}
$$

Eqs. (27) and (28) show that the regime of FLRW is diffusive.

In summary, if temporal effect is strong enough, the range $0<z \ll l_{2 D}$ can been split into two subrange: $0<$ $z \ll v / \gamma \ll l_{2 D}$ and $v / \gamma \ll z \ll l_{2 D}$. In the first subrange dynamical effect can be neglected and magnetic field line wandering presents ballistic process regardless of the strength of temporal effect. But in the second subrange the regime of FLRW can be transformed from ballistic into diffusive by temporal effect. The stronger temporal effect, i.e., the larger factor $\gamma$, leads to the longer subrange $v / \gamma \ll z \ll l_{2 D}$ and the smaller diffusion coefficient. Therefore, the temporal effect not only can reduce MSD but also change the regimes of magnetic field line wandering. However, for magnetostatic turbulence it is only ballistic in the whole range $0<z \ll l_{2 D}$. If the temporal effect is very weak so that $v / \gamma \gg l_{2 D}$, it is only ballistic in the whole range $0<z \ll l_{2 D}$ as same as in the magnetostatic case. 


\section{B. Dimensionless quantities in the range $\sigma \ll 2 l_{2 D}^{2} \ll 2 L_{2 D}^{2}$}

Taking the nondimensionlizing operation on Eq. (24), we can obtain

$$
\sigma^{\prime} \approx \frac{1}{2} R^{2}\left(z^{\prime}+e^{-z^{\prime}}-1\right)
$$

here the dimensionless quantities $z^{\prime}=\gamma z / v$ and $\sigma^{\prime}=\sigma /\left(2 l_{2 D}^{2}\right)$ are used. And we can see that a new dimensionless quantity occurs in the latter equation as follow

$$
R=\frac{\delta B_{2 D}}{B_{0}} \frac{v}{\gamma l_{2 D}}
$$

From Eq. (29) we can see that the dimensionless quantity $R$ controls FLRW in the range $\sigma \ll 2 l_{2 D}^{2} \ll 2 L_{2 D}^{2}$. In addition, the dimensionless mean square displacement $\sigma^{\prime}$ is proportional to the square of the dimensionless quantities $R$.

By employing the same nondimensionalizing method as above, the governing equation in the subrange $0<z \ll$ $v / \gamma \ll l_{2 D}$ (see Eq. (26) ) can be rewritten as

$$
\sigma^{\prime} \approx \frac{1}{4} R^{2} z^{\prime 2}
$$

and the governing equation in the subrange $v / \gamma \ll z \ll l_{2 D}$ (see Eq. (28)) can be nondimensionlized as

$$
\sigma^{\prime} \approx \frac{1}{2} R^{2}\left(z^{\prime}-1\right)
$$

From Eqs. (31) and (32) we can see that the dimensionless quantity $R$ controls the features of dimensionless mean square displacement $\sigma^{\prime}$ in the subranges $0<z \ll v / \gamma \ll l_{2 D}$ and $v / \gamma \ll z \ll l_{2 D}$, i.e., in the whole range $0<z \ll l_{2 D}$. Although it is ballistic in the former subrange and diffusive in the latter subrange, dimensionless mean square displacement $\sigma^{\prime}$ is always proportional to $R^{2}$ in the whole range $0<z \ll l_{2 D}$. Therefore, for the case $\sigma \ll 2 l_{2 D}^{2} \ll 2 L_{2 D}^{2}$ the dynamical effect of magnetic turbulence might have an impact on the regimes of FLRW, but it does not make any influence on the relationship between $\sigma^{\prime}$ and the new dimensionless quantity $R$. In other words, although the dynamical effect might change the regimes of FLRW from ballistic into diffusive, it has no any impact on the relations between $\sigma$ and the turbulence level $\delta B_{2 D} / B_{0}$.

\section{ANALYTICAL FORMULAS OF FIELD LINE WANDERING IN THE RANGE $2 l_{2 D}^{2} \ll 2 L_{2 D}^{2} \ll \sigma$}

In the range $2 l_{2 D}^{2} \ll 2 L_{2 D}^{2} \ll \sigma$, i.e., in the range outside box size, the following conditions must be satisfied

$$
\rho^{2}=\frac{\sigma}{2 l_{2 D}^{2}} \gg 1, \quad \rho^{2} \xi^{2}=\frac{\sigma}{2 L_{2 D}^{2}} \gg 1,
$$

here parameter $\xi=l_{2 D} / L_{2 D} \ll 1$ is used.

\section{A. The features in the range $2 l_{2 D}^{2} \ll 2 L_{2 D}^{2} \ll \sigma$}

By employing Eqs. (21) and (33), Eq. (14) can be rewritten as

$$
\begin{aligned}
\left(\frac{d \sigma}{d z}\right)^{2}= & 4 D(s, q) \frac{\delta B_{2 D}^{2}}{B_{0}^{2}} l_{2 D}^{2}\left(\frac{1-\xi^{q-1}}{q-1}+\frac{1}{s+1}\right) \\
& -2 D(s, q) \frac{\delta B_{2 D}^{2}}{B_{0}^{2}} l_{2 D}^{2} e^{-\frac{\gamma}{v} z}\left[\rho^{-2} \xi^{q-3} e^{-\rho^{2} \xi^{2}}-\rho^{-2} e^{-\rho^{2}}+\rho^{-2} e^{-\rho^{2}}\right] \\
& -2 D(s, q) \frac{\delta B_{2 D}^{2}}{B_{0}^{2}} l_{2 D}^{2} \frac{\gamma}{v} \int_{0}^{z} d z^{\prime} e^{-\frac{\gamma}{v} z^{\prime}}\left[\rho^{-2} \xi^{q-3} e^{-\rho^{2} \xi^{2}}-\rho^{-2} e^{-\rho^{2}}+\rho^{-2} e^{-\rho^{2}}\right] .
\end{aligned}
$$


From Eq. (33) we can find that $e^{-\rho^{2} \xi^{2}}$ and $e^{-\rho^{2}}$ all tend to zero in the range $2 l_{2 D}^{2} \ll 2 L_{2 D}^{2} \ll \sigma$. Then the latter equation can be simplified as

$$
\left(\frac{d \sigma}{d z}\right)^{2} \approx 4 D(s, q) \frac{\delta B_{2 D}^{2}}{B_{0}^{2}} l_{2 D}^{2}\left(\frac{1-\xi^{q-1}}{q-1}+\frac{1}{s+1}\right) .
$$

Since $\xi^{q-1} \ll 1$ for $q>1$, Eq. (35) becomes

$$
\left(\frac{d \sigma}{d z}\right)^{2} \approx 4 D(s, q) \frac{\delta B_{2 D}^{2}}{B_{0}^{2}} l_{2 D}^{2}\left(\frac{1}{q-1}+\frac{1}{s+1}\right) .
$$

By using the definition of diffusion coefficient of field line $\kappa_{F L T}=(1 / 2)(d \sigma / d z)$, we obtain

$$
\kappa_{F L T} \approx \sqrt{\frac{(q+1)(s-1)}{(q-1)(s+1)}} l_{2 D} \frac{\delta B_{2 D}}{B_{0}} .
$$

Since $\xi^{q-1} \gg 1$ for $-1<q<1$, by using the same method as above, from Eq. (35) we can deduce the diffusion coefficient as follow

$$
\kappa_{F L T} \approx \sqrt{\frac{(q+1)(s-1)}{(\boldsymbol{s}+\boldsymbol{q})(1-q)}} l_{2 D} \frac{\delta B_{2 D}}{B_{0}}\left(\frac{L_{2 D}}{l_{2 D}}\right)^{\frac{1-q}{2}} .
$$

We can find that Eqs. (37) and (38) are perfectly identical with Eqs. (35) and (36) in the article of Shalchi (2011) which are from magnetostatic model, respectively. Therefore, we can see that in the range larger than the box size the diffusion coefficients are independent of the temporal effect irrespective of the strength of temporal effect. For this result we can obtain some qualitative explanation from Eq. (13). The first term on the right hand side of Eq. (13) is independent of temporal effect, while the second term and third terms are related to time $t$, that is, related to position $z$. For the limit $\sigma(z) \rightarrow \infty$ corresponding to $z \rightarrow \infty$, then $e^{-v z / \gamma} \rightarrow 0$, so the second and third terms of Eq. (13) can be ignored in comparison with the first term. That is, only the first term in Eq. (13) is remained for $z \rightarrow \infty$. Therefore, for $z \rightarrow \infty$ the regime of particle transport tends to the magnetostatic result, i.e., diffusion (see Shalchi 2011).

\section{B. Dimensionless parameters in the range $2 l_{2 D}^{2} \ll 2 L_{2 D}^{2} \ll \sigma$}

By using the same method as in the subsection V.B and employing the dimensionless quantity $R=\left(\delta B_{2 D} v\right) /\left(B_{0} \gamma l_{2 D}\right)$, from Eqs. (37) and (38) we can get the following dimensionless equations

$$
\begin{array}{ll}
\kappa_{F L T}^{\prime} \approx \frac{1}{2} \sqrt{\frac{(q+1)(s-1)}{(q-1)(s+1)}} R, & \text { for } \quad q>1 \\
\kappa_{F L T}^{\prime} \approx \frac{1}{2} \sqrt{\frac{(q+1)(s-1)}{(1-q)(s+q)}} R \xi^{(q-1) / 2}, & \text { for } \quad-1<q<1
\end{array}
$$

where the dimensionless diffusion coefficient is defined as $\kappa_{F L T}^{\prime}=(1 / 2)\left(d \sigma^{\prime} / d z^{\prime}\right)$. From Eqs. (39) and (40) we can see that the dimensionless diffusion coefficient $\kappa_{F L T}^{\prime}$ is proportional to dimensionless parameter $R$ for any allowed value of $q$ in the range $2 l_{2 D}^{2} \ll 2 L_{2 D}^{2} \ll \sigma$. In other words, the diffusion coefficient $\kappa_{F L T}$ is proportional to $\delta B_{2 D} / B_{0}$ in the range $2 l_{2 D}^{2} \ll 2 L_{2 D}^{2} \ll \sigma$ regardless of the energy index $q$.

\section{ANALYTICAL FORMULAS OF FIELD LINE WANDERING IN THE RANGE $2 l_{2 D}^{2} \ll \sigma \ll 2 L_{2 D}^{2}$}

We can obtain the conditions $\rho^{2}=\sigma /\left(2 l_{2 D}^{2}\right) \gg 1$ and $\rho^{2} \xi^{2}=\sigma /\left(2 L_{2 D}^{2}\right) \ll 1$ in the range $2 l_{2 D}^{2} \ll \sigma \ll 2 L_{2 D}^{2}$. By using Eq. (21) we can simplify Eq. (14) as follow

$$
\begin{aligned}
\left(\frac{d \sigma}{d z}\right)^{2} \approx & 4 D(s, q) \frac{\delta B_{2 D}^{2}}{B_{0}^{2}} l_{2 D}^{2}\left(\frac{1-\xi^{q-1}}{q-1}+\frac{1}{s+1}\right) \\
& -2 D(s, q) \frac{\delta B_{2 D}^{2}}{B_{0}^{2}} l_{2 D}^{2} e^{-\frac{\gamma}{v} z}\left[\rho^{1-q} \Gamma\left(\frac{q-1}{2}\right)-\frac{2}{q-1} \xi^{q-1}+\frac{2}{q+1} \rho^{2} \xi^{q+1}+\rho^{-2} e^{-\rho^{2}}\right] \\
& -2 D(s, q) \frac{\delta B_{2 D}^{2}}{B_{0}^{2}} l_{2 D}^{2} \frac{\gamma}{v} \int_{0}^{z} d z^{\prime} e^{-\frac{\gamma}{v} z^{\prime}}\left[\rho^{1-q} \Gamma\left(\frac{q-1}{2}\right)-\frac{2}{q-1} \xi^{q-1}+\frac{2}{q+1} \rho^{2} \xi^{q+1}+\rho^{-2} e^{-\rho^{2}}\right] .
\end{aligned}
$$




\section{A. The special case $q>1$}

For $q>1$ the second and third terms on the right hand side of Eq. (41) can be neglected comparing to the first term. Then Eq. (41) can be simplified as

$$
\left(\frac{d \sigma}{d z}\right)^{2} \approx 4 D(s, q) \frac{\delta B_{2 D}^{2}}{B_{0}^{2}} l_{2 D}^{2}\left(\frac{1}{q-1}+\frac{1}{s+1}\right) .
$$

From the latter equation we can get the diffusion coefficient of magnetic field line wandering as

$$
\kappa_{F L T} \approx \sqrt{\frac{(q+1)(s-1)}{(q-1)(s+1)}} \frac{\delta B_{2 D}}{B_{0}} l_{2 D}
$$

which coincides with Eq. (29) in Shalchi (2011) for the same special case. Through nondimensionalizing of Eq. (43) we can obtain

$$
\kappa_{F L T}^{\prime} \approx \frac{1}{2} \sqrt{\frac{(q+1)(s-1)}{(q-1)(s+1)}} R
$$

where the dimensionless parameter $R=\left(\delta B_{2 D} v\right) /\left(B_{0} \gamma l_{2 D}\right)$ occurs again. Therefore, for this special case the dimensionless diffusion coefficient of FLRW is proportional to the new dimensionless parameter $R$, or diffusion coefficient $\kappa_{F L T}$ is proportional to turbulence level $\delta B_{2 D} / B_{0}$.

\section{B. The special case $-1<q<1$}

For $-1<q<1$ Eq. (14) can be simplifies as

$$
\begin{aligned}
\left(\frac{d \sigma}{d z}\right)^{2} \approx & 4 D(s, q) \frac{\delta B_{2 D}^{2}}{B_{0}^{2}} l_{2 D}^{2}\left(\frac{\xi^{q-1}}{1-q}+\frac{1}{s+1}\right) \\
& -2 D(s, q) \frac{\delta B_{2 D}^{2}}{B_{0}^{2}} l_{2 D}^{2} e^{-\frac{\gamma}{v} z}\left[\rho^{1-q} \Gamma\left(\frac{q-1}{2}\right)-\frac{2}{q-1} \xi^{q-1}+\frac{2}{q+1} \rho^{2} \xi^{q+1}\right] \\
& -2 D(s, q) \frac{\delta B_{2 D}^{2}}{B_{0}^{2}} l_{2 D}^{2} \frac{\gamma}{v} \int_{0}^{z} d z^{\prime} e^{-\frac{\gamma}{v} z^{\prime}}\left[\rho^{1-q} \Gamma\left(\frac{q-1}{2}\right)-\frac{2}{q-1} \xi^{q-1}+\frac{2}{q+1} \rho^{2} \xi^{q+1}\right]
\end{aligned}
$$

By taking the derivative of the latter equation over $z$ we can obtain

$$
\frac{d^{2} \sigma}{d z^{2}} \approx \frac{D(s, q)}{2} \Gamma\left(\frac{q+1}{2}\right) \frac{\delta B_{2 D}^{2}}{B_{0}^{2}} e^{-\frac{\gamma}{v} z}\left(\frac{\sigma}{2 l_{2 D}^{2}}\right)^{-\frac{1+q}{2}}
$$

By nondimensionalizing the latter equation we can obtain

$$
\frac{d^{2} \sigma^{\prime}}{d z^{\prime 2}} \approx \frac{D(s, q)}{4} \Gamma\left(\frac{q+1}{2}\right) e^{-z^{\prime}} \sigma^{\prime-\frac{1+q}{2}} R^{2} .
$$

From the latter equation we can see that the dimensionless quantity $R$ controls the properties of magnetic field line wandering for the special case $-1<q<1$. We set the following relationship

$$
\sigma^{\prime}=h(R) p\left(z^{\prime}\right)
$$

Inserting Eq. (48) into Eq. (47) we can obtain

$$
h(R) \frac{d^{2} p\left(z^{\prime}\right)}{d z^{\prime 2}} \approx \frac{D(s, q)}{4} \Gamma\left(\frac{q+1}{2}\right) e^{-z^{\prime}} h(R)^{-\frac{1+q}{2}} p\left(z^{\prime}\right)^{-\frac{1+q}{2}} R^{2} .
$$

Thus we can find the following formula

$$
h(R) \propto R^{2} h(R)^{-(1+q) / 2},
$$


or

$$
h(R) \propto R^{4 /(3+q)}
$$

Therefore, from Eq. (48) we can obtain

$$
\sigma^{\prime} \approx h(R) p\left(z^{\prime}\right) \propto R^{4 /(3+q)} p\left(z^{\prime}\right)
$$

From the above discussion we can see that the relationship (52) is irrelevant to temporal effect. So the dynamical effect has no impact on the relationship between the dimensionless mean square displacement $\sigma^{\prime}$ and the dimensionless quantity $R$.

\section{Simplification of the governing equation for $-1<q<1$ in the range $2 l_{2 D}^{2} \ll \sigma \ll 2 L_{2 D}^{2}$}

Let's set $\sigma=g(z) e^{f(z)}$, the governing equation for $-1<q<1$ in the range $2 l_{2 D}^{2} \ll \sigma \ll 2 L_{2 D}^{2}$ (see Eq. (46)) can be simplified as

$$
\left[\frac{d^{2} g}{d z^{2}}+2 \frac{d g}{d z} \frac{d f}{d z}+g\left(\frac{d f}{d z}\right)^{2}+g \frac{d^{2} f}{d z^{2}}\right] e^{f}=\frac{D(s, q)}{2^{(1-q) / 2}} \frac{\delta B_{2 D}^{2}}{B_{0}^{2}} \Gamma\left(\frac{q+1}{2}\right) l_{2 D}^{1+q} g^{-\frac{1+q}{2}} e^{-\frac{\gamma}{v} z} e^{-\frac{1+q}{2} f}
$$

By comparing the left-hand side of the latter equation with the right-hand side, we can get the following equations

$$
f=-\frac{2}{3+q} \frac{\gamma}{v} z
$$

and

$$
\frac{d^{2} g}{d z^{2}}+2 \frac{d g}{d z} \frac{d f}{d z}+g\left(\frac{d f}{d z}\right)^{2}+g \frac{d^{2} f}{d z^{2}}=\frac{D(s, q)}{2^{(1-q) / 2}} \frac{\delta B_{2 D}^{2}}{B_{0}^{2}} \Gamma\left(\frac{q+1}{2}\right) l_{2 D}^{1+q} g^{-\frac{1+q}{2}}
$$

From Eqs. (54) and (55) we can obtain the equation of $g(z)$ as

$$
\frac{d^{2} g}{d z^{2}}-\frac{4}{3+q} \frac{\gamma}{v} \frac{d g}{d z}+\left(\frac{2}{3+q} \frac{\gamma}{v}\right)^{2} g=\frac{D(s, q)}{2^{(1-q) / 2}} \frac{\delta B_{2 D}^{2}}{B_{0}^{2}} \Gamma\left(\frac{q+1}{2}\right) l_{2 D}^{1+q} g^{-\frac{1+q}{2}}
$$

The latter equation can be rewritten as

$$
\frac{d^{2} g}{d z^{2}}-2 B \frac{d g}{d z}+B^{2} g-A g^{-\frac{1+q}{2}}=0
$$

with

$$
\begin{aligned}
A & =\frac{D(s, q)}{2^{(1-q) / 2}} \frac{\delta B_{2 D}^{2}}{B_{0}^{2}} \Gamma\left(\frac{q+1}{2}\right) l_{2 D}^{1+q} \\
B & =\frac{2}{3+q} \frac{\gamma}{v}
\end{aligned}
$$

By employing Eq. (54) the mean square displacement of FLRW can be shown as

$$
\sigma(z)=g(z) e^{-\frac{2}{3+q} \frac{\gamma}{v} z}
$$

where $g(z)$ is the solution of Eq. (57). Since the mean square displacement $\sigma(z)$ monotonically increases with position $z$, but $e^{-2 \gamma z /((3+q) v)}$ monotonically decreases with $z$, so $g(z)$ should be the monotonically increasing function of position $z$. In what follows, we explore some special cases of Eq. (57). 


\section{The case $B=0$}

For the case $B=0$ corresponding to magnetostatic turbulence, Eq. (57) can be simplified as

$$
\frac{d^{2} g}{d z^{2}}=A g^{-\frac{1+q}{2}}
$$

By setting $g=\alpha|z|^{\beta}$ and using the relationship $d g / d z=\alpha \beta|z|^{\beta-1}$ and $d^{2} g / d z^{2}=\alpha \beta(\beta-1)|z|^{\beta-2}$, the Eq. (61) can be rewritten as

$$
\alpha \beta(\beta-1)|z|^{\beta-2}=A \alpha^{-\frac{1+q}{2}}|z|^{-\frac{1+q}{2} \beta} .
$$

From Eq. (62) we can obtain the formulas of $\alpha$ and $\beta$ as follows

$$
\begin{aligned}
& \alpha=\left[\frac{(3+q)^{2}}{4(1-q)} \frac{D(s, q)}{2^{(1-q) / 2}} \frac{\delta B_{2 D}^{2}}{B_{0}^{2}} \Gamma\left(\frac{q+1}{2}\right) l_{2 D}^{1+q}\right]^{\frac{2}{3+q}} \\
& \beta=\frac{4}{3+q} .
\end{aligned}
$$

By combining Eqs. (63) and (64) one can get the mean square displacement of magnetostatic turbulence as

$$
\sigma=\left[\frac{(3+q)^{2}}{(1-q)} \frac{D(s, q)}{2^{(5-q) / 2}} \frac{\delta B_{2 D}^{2}}{B_{0}^{2}} \Gamma\left(\frac{q+1}{2}\right) l_{2 D}^{1+q}\right]^{\frac{2}{3+q}}|z|^{\frac{4}{3+q}}
$$

The latter formula is in agreement with previous analytical result derived in Shalchi (2011).

\section{E. The case $B=\varepsilon$}

If $B$ is a very small quantity, perturbation method (see, e.g., Paulsen 2014) can be used to treat Eq. (57). Expanding $g(z)$ as a series of $\varepsilon$

$$
g=g_{0}+\varepsilon g_{1}+\varepsilon^{2} g_{2}+\varepsilon^{3} g_{3}+\cdots \cdots
$$

and inserting Eq. (66) into Eq. (57), one can achieve the following equations

$$
\begin{aligned}
& \varepsilon^{0}: \frac{d^{2} g_{0}}{d z^{2}}=A g_{0}^{-\frac{1+q}{2}} \\
& \varepsilon^{1}: \frac{d^{2} g_{1}}{d z^{2}}-2 \frac{d g_{0}}{d z}=-\frac{1+q}{2} A \frac{g_{1}}{g_{0}^{(3+q) / 2}} \\
& \varepsilon^{2}: \frac{d^{2} g_{2}}{d z^{2}}-2 \frac{d g_{1}}{d z}+g_{0}=A g_{0}^{-(1+q) / 2}\left[-\frac{1+q}{2} \frac{g_{2}}{g_{0}}+\frac{(1+q)(3+q)}{8}\left(\frac{g_{1}}{g_{0}}\right)^{2}\right]
\end{aligned}
$$

and

$$
\varepsilon^{3}: \frac{d^{2} g_{3}}{d z^{2}}-2 \frac{d g_{2}}{d z}+g_{1}=A g_{0}^{-(1+q) / 2}\left[-\frac{1+q}{2} \frac{g_{3}}{g_{0}}+\frac{(1+q)(3+q)}{4} \frac{g_{1} g_{2}}{g_{0}}-\frac{(1+q)(3+q)(5+q)}{48}\left(\frac{g_{1}}{g_{0}}\right)^{3}\right]
$$

and so on.

We can easily obtain the solution of Eq. (67) as

$$
g_{0}(z)=\left[\frac{(3+q)^{2}}{(1-q)} \frac{D(s, q)}{2^{(5-q) / 2}} \frac{\delta B_{2 D}^{2}}{B_{0}^{2}} \Gamma\left(\frac{q+1}{2}\right) l_{2 D}^{1+q}\right]^{\frac{2}{3+q}}|z|^{\frac{4}{3+q}}
$$

which corresponds to magnetostatic case.

The solution of Eq. (68) can be found in Appendix, here we directly show it as the following

$$
\begin{array}{lrl}
g_{1}=c_{1} z^{2(1+q) /(3+q)}+c_{2} z^{(1-q) /(3+q)}+b_{0} z^{(7+q) /(3+q)}, & \text { for } & q \neq-1 / 3 \\
g_{1}=\left(c_{1}+c_{2} \ln z\right) \sqrt{z}+b_{0} z^{(7+q) /(3+q)} . & \text { for } & q=-1 / 3
\end{array}
$$


And using the same technique the solutions of higher-order equations can also be obtained successively.

By combining the solutions of all order equations we can get the general solution of Eq. (57) as follow

$$
\sigma=\left(g_{0}+\varepsilon g_{1}+\varepsilon^{2} g_{2}+\varepsilon^{3} g_{3}+\cdots \cdots\right) e^{-\frac{2}{3+q} \frac{\gamma}{v} z} .
$$

For $\varepsilon=0$ we can get the solution corresponding to magnetostatic turbulence. And if $\varepsilon \neq 0$, the terms $\varepsilon z^{(7+q) /(3+q)}, \ldots \ldots$ occur on the right-hand side of Eq. (74). So MSD is a complicated function of $z$, and only according to the formula (74) we cannot find the regimes of FLRW. In what follows, the possible regimes of FLRW and the influence of temporal effect on the regimes will be explored.

For $\gamma=0$ corresponding to magnetostatic case, the power law exponent $\beta$ is equal to $4 /(3+q)$ which is the well known result in diffusion theory of FLRW (see Shalchi \& Kourakis, 2007a). For the case that $\gamma$ tends to $\infty$, it is more convenient to directly investigate Eq. (46). And if temporal factor $\gamma$ tends to infinity we can find that the right hand side of Eq. (46) tends to zero. Therefore, for this case FLRW tends to diffusion, i.e., $\beta$ tends to 1 . In the following we explore the case of $\gamma \neq 0, \infty$.

Firstly, since the right-hand side of Eq. (46) is greater than zero for $\gamma \neq 0, \infty$, so the left-hand side is also greater than zero, i.e., $d^{2} \sigma / d z^{2}>0$. So Eq. (46) only describe superdiffusive process. Secondly, considering that $\sigma$ is the function of temporal factor $\gamma$, we explore the variation rule of $\sigma$ with $\gamma$. After taking derivative of Eq. (46) over $\gamma$ we can obtain

$$
\frac{d^{3} \sigma}{d z^{2} d \gamma}=\frac{D(s, q)}{2} \Gamma\left(\frac{q+1}{2}\right) \frac{\delta B_{2 D}^{2}}{B_{0}^{2}}\left(2 l_{2 D}^{2}\right)^{(1+q) / 2} e^{-\gamma z / v} \sigma^{-(3+q) / 2}\left(-\frac{z}{v} \sigma-\frac{1+q}{2} \frac{d \sigma}{d \gamma}\right) .
$$

Because $d^{2} \sigma / d z^{2}>0$, obviously, only $d \sigma / d \gamma<0$ satisfies the latter equation. That is, temporal effect reduces MSD of FLRW.

To summarize the above discussion, FLRW is superdiffusive for the case $\gamma<\infty$, and it tends to diffusive if $\gamma$ tends to infinity. But subdiffusion does not occur. So temporal effect could change the regimes of FLRW from superdiffusion into diffusion. From Eq. (74) we can find that MSD of FLRW with dynamical effect is no longer the simple form $\sigma=\alpha|z|^{\beta}$ or the logarithmic form $\sigma \sim z \ln z$ (see Kourakis et al. 2009). Thus we can see that the temporal effect not only changes the specific form of MSD but also affects the diffusion regimes of FLRW. In fact, this is not a new result. Some previous papers already have found this effect (see Shalchi 2010a, Guest \& Shalchi 2012).

\section{F. The condition of neglecting the term $A^{-(1+q) / 2}$ in equation $d^{2} g / d z^{2}-2 B d g / d z+B^{2} g-A g-(1+q) / 2=0$}

Eq. (56) can be nondimensionlized as the following

$$
\frac{d^{2} g^{\prime}}{d z^{\prime 2}}-\frac{4}{3+q} \frac{d g^{\prime}}{d z^{\prime}}+\left(\frac{2}{3+q}\right)^{2} g^{\prime}-\frac{1}{4} \frac{D(s, q) \Gamma\left(\frac{q+1}{2}\right)}{g^{\prime(1+q) / 2}} R^{2}=0
$$

with $g^{\prime}=g /\left(2 l_{2 D}^{2}\right), z^{\prime}=\gamma z / v$, and $R=\left(\delta B_{2 D} v\right) /\left(B_{0} \gamma l_{2 D}\right)$. Here we can see that the dimensionless quantity $R$ controls the physical process described by the latter equation. If dimensionless quantity $R$ is mall enough or $g^{\prime}=g /\left(2 l_{2 D}^{2}\right)$ is large enough, the inhomogeneous equation can be simplified down to homogeneous equation. In what follows, we start from Eq. (57) to explore the condition that the term $\mathrm{Ag}^{-(1+q) / 2}$ can be ignored.

If the fourth term $A g^{-(1+q) / 2}$ on the left-hand side of Eq. (57) is much less than the other terms $d^{2} g / d z^{2}, 2 B d g / d z$ and $B^{2} g$, the term $A g^{-(1+q) / 2}$ can be neglected. Then we can get

$$
\frac{d^{2} g}{d z^{2}}-2 B \frac{d g}{d z}+B^{2} g=0
$$

The general solution of the latter equation can be obtained as

$$
g=\left(c_{1}+c_{2} z\right) e^{B z}
$$

Then MSD can be written as

$$
\sigma=g e^{f}=c_{1}+c_{2} z
$$

If we set $\sigma(z=0)=0, c_{1}$ is equal to zero. Since $\sigma(z)$ is positive, parameter $c_{2}$ also should be positive. From the latter equation we can see that Eq. (177) describes diffusion process. 
By employing Eq. (78) the formulae $d^{2} g / d z^{2}, 2 B d g / d z$ and $B^{2} g$ in Eq. (77) can be obtained as follows

$$
\begin{aligned}
\frac{d^{2} g}{d z^{2}} & =2 c_{2} B e^{B z}+\left(c_{1}+c_{2} z\right) B^{2} e^{B z}, \\
2 B \frac{d g}{d z} & =2 c_{2} B e^{B z}+2\left(c_{1}+c_{2} z\right) B^{2} e^{B z} \\
B^{2} g & =\left(c_{1}+c_{2} z\right) B^{2} e^{B z} .
\end{aligned}
$$

Comparing Eqs. (80)-(82), since $c_{1}=0$ and $c_{2}>0$ and $B>0$ we can find that $B^{2} g$ is smaller than $d^{2} g / d z^{2}, 2 B d g / d z$. Therefore, if the term $\mathrm{Ag}^{-(1+q) / 2}$ in Eq. (57) could be neglected, the following condition should be satisfied

$$
B^{2} g \gg A g^{-(1+q) / 2} .
$$

By using formula $\sigma=g(z) e^{f(z)}$ we can rewrite Eq. (83) as

$$
\sigma \gg\left(\frac{A}{B^{2}}\right)^{2 /(3+q)} e^{f},
$$

which is the condition that the term $A g^{-(1+q) / 2}$ in equation $d^{2} g / d z^{2}-2 B d g / d z+B^{2} g-A g^{-(1+q) / 2}=0$ can be neglected.

\section{G. Summary for $-1<q<1$ in the range $2 l_{2 D}^{2} \ll \sigma \ll 2 L_{2 D}^{2}$}

In this section we explore the properties of FLRW for $-1<q<1$ in the range $2 l_{2 D}^{2} \ll \sigma \ll 2 L_{2 D}^{2}$. From Eq. (84) the following formula can be obtained

$$
\left(\frac{A}{B^{2}}\right)^{2 /(3+q)} e^{f} \ll \sigma \ll 2 L_{2 D}^{2}
$$

By combining Eqs. (58) and (59) the latter formula can be written as

$$
\left[\frac{D(s, q)}{2^{(1-q) / 2}} \frac{\delta B_{2 D}^{2}}{B_{0}^{2}} \frac{v^{2}}{\gamma^{2}} \Gamma\left(\frac{q+1}{2}\right) l_{2 D}^{1+q}\right]^{\frac{2}{3+q}}\left(\frac{3+q}{2}\right)^{\frac{4}{3+q}} \ll 2 L_{2 D}^{2} e^{\frac{2}{3+q} \frac{\gamma}{v} z} .
$$

By using nondimensionlizing method formula (86) can be rewritten as,

$$
\left(\frac{3+q}{4}\right)^{\frac{4}{3+q}}\left[D(s, q) \Gamma\left(\frac{q+1}{2}\right)\right]^{\frac{2}{3+q}} R^{\frac{4}{3+q}} \xi^{2} \ll e^{\frac{2}{3+q} \chi}
$$

where we use the following dimensionless quantities

$$
\chi=\frac{\gamma}{v} L_{2 D}, \quad \xi=\frac{l_{2 D}}{L_{2 D}}, \quad R=\frac{\delta B_{2 D}}{B_{0}} \frac{v}{\gamma l_{2 D}} .
$$

Obviously, from the latter inequality we can see that the dimensionless quantities $R, \xi$ and $\chi$ determine whether the term on right-hand side of Eq. (57) can be ignored or not. When dimensionless quantities $\chi$ and $\xi$ are assumed constants and $R$ is small enough, inequality (87) could hold. Similarly, when $\chi$ and $R$ are fixed and $\xi$ is small enough, or when $\xi$ and $R$ are fixed and $\chi$ is large enough, inequality (87) also could hold.

In the previous paragraphs by using nondimensionlizing method we explore the condition that inequality (87) holds. In what follows, we investigate the same issue by directly using the pertinent physical quantities in real space. Let us suppose that the inertial range index $s$, the energy range index $q$, bend-over scale $l_{2 D}$, box scale $L_{2 D}$ and turbulence level $\delta B_{2 D} / B_{0}$ are all constants, and if temporal factor $\gamma$ is large enough, inequality (87) could hold. If we set the quantities $s, q, l_{2 D}, L_{2 D}$, and $\gamma$ as constants, inequality (87) is established as long as $\delta B_{2 D} / B_{0}$ is sufficiently small. On the other hand, if the quantities $s, q, L_{2 D}, \delta B_{2 D} / B_{0}$, and $\gamma$ are constants, but the bend-over scale $l_{2 D}$ is small enough, or the quantities $s, q, l_{2 D}, \delta B_{2 D} / B_{0}$, and $\gamma$ are constants, but the box scale is large enough, inequality (87) could also be established. Therefore, not only the energy index $q$ and temporal effect could change the regimes of FLRW, but also the bendover scale $l_{2 D}$, box size $L_{2 D}$, turbulence level $\delta B_{2 D} / B_{0}$ also could affect the regimes of FLRW. In fact, for a certain magnetic turbulence system the physical quantities $s, q, l_{2 D}, L_{2 D}, \delta B_{2 D} / B_{0}$, and $\gamma$ are probably dependent of each other. So the number of quantities that could affect independently the regimes of FLRW might be less than 6 . 


\section{SUMMARY AND CONCLUSION}

In the present article, by using field line tracing method we have investigated analytically the properties of FLRW in all possible length scales. For simplification only the simple damping dynamical model $\Gamma(\vec{k}, t)=e^{-\gamma t}$ with constant factor $\gamma$ is employed. The model $\Gamma(\vec{k}, t)=e^{-\gamma t}$ denotes that the temporal correlation function decay exponentially. Of course, as a matter of fact, temporal factor $\gamma$ should be the function of wave number $k$ and Alfvén speed $v_{A}$ and so on (see, e.g., Shalchi 2010a, Guest \& Shalchi 2012). For the purpose of simplification in this article we assume temporal factor $\gamma$ is a constant and leave the variable factor $\gamma$ for the future task. By the investigation and discussion in this article we find that if temporal effect is strong enough it can affect the field line wandering in the range $\sigma \ll 2 l_{2 D}^{2} \ll 2 L_{2 D}^{2}$, and the energy range spectral index $q$ determines the properties of field line wandering in the range $2 l_{2 D}^{2} \ll 2 L_{2 D}^{2} \ll \sigma$ and for the case $q>1$ in the range $2 l_{2 D}^{2} \ll \sigma \ll 2 L_{2 D}^{2}$, but for the case $-1<q<1$ in the range $2 l_{2 D}^{2} \ll \sigma \ll 2 L_{2 D}^{2}$ both $\gamma$ and $q$ influence the field line wandering. In addition, we obtain the following results.

(1) In order to describe the properties of FLRW of pure $2 \mathrm{D}$ turbulence with damping dynamical model a new dimensionless parameter $R=\left(\delta B_{2 D} v\right) /\left(B_{0} \gamma l_{2 D}\right)$ is needed to be introduced. For pure 2D turbulence Kubo number does not exist, but $R$ does.

(2) If the temporal effect is strong enough, it could change the transport regimes from ballistic process into diffusive one in the range $\sigma \ll 2 l_{2 D}^{2} \ll 2 L_{2 D}^{2}$. In the range $2 l_{2 D}^{2} \ll \sigma \ll 2 L_{2 D}^{2}$ the temporal effect could change the transport regimes from superdiffusion into diffusion but without subdiffusion. However, the temporal effect does not influence FLRW in the range $2 l_{2 D}^{2} \ll 2 L_{2 D}^{2} \ll \sigma$ regardless of the strength of temporal effect. That is, temporal effect has no any impact on FLRW in the range outside box size.

(3) Dimensionless mean square displacement $\sigma^{\prime}$ is in proportion to $R^{2}$ in the range $\sigma \ll 2 l_{2 D}^{2} \ll 2 L_{2 D}^{2}$. And $\sigma^{\prime}$ is in linear relation with $R^{4 /(3+q)}$ for the case $-1<q<1$ in the range $2 l_{2 D}^{2} \ll \sigma \ll 2 L_{2 D}^{2}$. But $\sigma^{\prime}$ and $R$ are in the direct ratio for the case $q>1$ in the range $2 l_{2 D}^{2} \ll \sigma \ll 2 L_{2 D}^{2}$ and for any allowed value $q$ in the range $2 l_{2 D}^{2} \ll 2 L_{2 D}^{2} \ll \sigma$. Of course, there are the same relationship as listed above between mean square displacement $\sigma$ and turbulence level $\delta B_{2 D} / B_{0}$. Although temporal effect can reduce FLRW and even change the regimes of FLRW, it does not affect the relationship between dimensionless mean square displacement $\sigma^{\prime}$ and dimensionless quantity $R$ in all possible length scales. That is, temporal effect does not affect the relationship between mean square displacement $\sigma$ and turbulence level $\delta B_{2 D} / B_{0}$.

The dimensionless quantity $R$ introduced in this article is related to temporal effect of turbulence. In the future we will use real space method and the Unified NonLinear Transport (UNLT) theory (see Shalchi 2010b) and so on to dig more deeply into the features of the new dimensionless quantity $R$.

In this paper, we concentrate on the effects of the temporal factor and energy range index on the regimes of field line wandering. However, other physical properties such as turbulence level, bend-over scale, and box-scale might also have their effects. In addition, we only explore field line wandering for damping model with constant temporal factor. But the temporal factor might be the function of wave number and Alfvén wave speed and so on (see, e.g., Shalchi et al. 2007; Shalchi 2010a; Guest \& Shalchi 2012). Therefore, our results might be oversimplified. Furthermore, the properties of the field line wandering with other dynamical models, e.g., plasma wave model, sweeping damping model, etc, are also important research topics. Moreover, the influence of dynamical effect on energetic charged particle's transport is another key problem worth paying attention. Finally, the dimensionless quantities corresponding to the above physical problems also need to be explored carefully. We will explore the problems listed above in the future work.

\section{ACKNOWLEDGMENTS}

We are partly supported by grants NNSFC 41125016, NNSFC 41574172, and NNSFC 41374177.

\section{Appendix A SOLVING THE FIRST-ORDER EQ. (68)}

Substituting Eq. (71) into Eq. (68), we get

$$
\frac{d^{2} g_{1}}{d z^{2}}+\frac{2\left(1-q^{2}\right)}{(3+q)^{2}} g_{1} z^{-2}=\left[\frac{D(s, q)}{2} \frac{\delta B_{2 D}^{2}}{B_{0}^{2}} \Gamma\left(\frac{q+1}{2}\right)\left(2 l_{2 D}^{2}\right)^{(1+q) / 2} \frac{(3+q)^{2}}{4(1-q)}\right]^{2 /(3+q)} \frac{8}{3+q} z^{(1-q) /(3+q)}
$$

The latter equation can be rewritten as

$$
\frac{d^{2} g_{1}}{d z^{2}}+M z^{-2} g_{1}=N z^{(1-q) /(3+q)}
$$


where we introduce the parameters

$$
\begin{gathered}
M=\frac{2\left(1-q^{2}\right)}{(3+q)^{2}}, \\
N=\left[\frac{D(s, q)}{2} \frac{\delta B_{2 D}^{2}}{B_{0}^{2}} \Gamma\left(\frac{q+1}{2}\right)\left(2 l_{2 D}^{2}\right)^{(1+q) / 2} \frac{(3+q)^{2}}{4(1-q)}\right]^{2 /(3+q)} \frac{8}{3+q} .
\end{gathered}
$$

Eq. (A-2) is a Euler equation and the solution for $q \neq-1 / 3$ is as

$$
g_{1}=c_{1} e^{r_{1} t}+c_{2} e^{r_{2} t}+b_{0} e^{t(7+q) /(3+q)}=c_{1} z^{r_{1}}+c_{2} z^{r_{2}}+b_{0} z^{(7+q) /(3+q)},
$$

here the following parameters are used

$$
b_{0}=\frac{N}{\left(\frac{7+q}{3+q}\right)^{2}-\left(\frac{7+q}{3+q}\right)+M}
$$

and for $q>-1 / 3$

$$
\begin{aligned}
& r_{1}=\frac{2(1+q)}{3+q}, \\
& r_{2}=\frac{1-q}{3+q}
\end{aligned}
$$

for $-1<q<-1 / 3$

$$
\begin{aligned}
& r_{1}=\frac{1-q}{3+q}, \\
& r_{2}=\frac{2(1+q)}{3+q} .
\end{aligned}
$$

And for $q=-1 / 3$ we can get the general solution

$$
g_{1}=\left(c_{1}+c_{2} \ln z\right) \sqrt{z}+b_{0} z^{(7+q) /(3+q)} .
$$

The specific expressions of $c_{1}, c_{2}$ can be determined by using the specific conditions, e.g., initial conditions, etc. For simplification we only consider the cases for the energy index $-1<q<1$ in the above discussion because for $q>1$ some complicated cases of multiple roots occur.

[1] J. R. Jokipii and E. N. Parker, Astrophys. J. 155, 799 (1969).

[2] J. Giacalone and J. R. Jokipii, J. Geophys. Res. 101, 11095 (1996).

[3] B. D. G. Chandran and S. C. Cowley, Phys. Rev. Lett. 80, 3077 (1998).

[4] A. Lazarian, Astrophys. J. 645, L25 (2006).

[5] J. Skilling, I. McIvor, and J. A. Holmes, MNRAS 167, 87 (1974).

[6] J. Giacalone and J. R. Jokipii, Astrophys. J. 520, 204 (1999).

[7] J. Kóta and J. R. Jokipii, Astrophys. J. 531, 1067 (2000).

[8] W. H. Matthaeus, G. Qin, J. W. Bieber, and G. P. Zank, Astrophys. J. 590, L53 (2003).

[9] A. Shalchi and I. Kourakis, Phys. Plasmas 14, 092903 (2007a).

[10] A. Shalchi and I. Kourakis, Phys. Plasmas 14, 112901 (2007b).

[11] A. Shalchi, R. C. Tautz, and R. Schlickeiser, Astron. Astrophys. 475, 415 (2007).

[12] R. Schlickeiser, Cosmic Ray Astrophysics (Springer, Berlin, 2002).

[13] G. M. Webb, G. P. Zank, E. Kh. Kaghashvili, and J. A. le Roux, Astrophys. J. 651, 211 (2006).

[14] G. Qin, Astrophys. J. 656, 217 (2007).

[15] A. Shalchi and I. Kourakis, Astron. Astrophys. 470, 405 (2007c).

[16] R. C. Tautz, A. Shalchi, and R. Schlickeiser, Astrophys. J. 672, 642 (2008).

[17] A. Shalchi, G. M. Webb, J. A. le Roux, and G. P. Zank, Ap\&SS 321, 197 (2009). 
[18] B. Weinhorst and A. Shalchi, MNRAS 406, 634 (2010).

[19] G. M. Webb, E. Kh. Kaghashvili, J. A. le Roux, A. Shalchi, G. P. Zank, and G. Li., J. Phys. A: Math. Theor. 42, 235502 (2009).

[20] K. Buffie and A. Shalchi, Ap\&SS 340, 351 (2012).

[21] G. Qin and L.-H. Zhang, Astrophys. J. 787, 12 (2014).

[22] J. R. Jokipii, Astrophys. J. 146, 480 (1966).

[23] A Shalchi, Phys. Plasmas 22, 010704 (2015).

[24] A. Shalchi, Nonlinear Cosmic Ray Diffusion (Springer, Berlin, 2009).

[25] W. H. Matthaeous, P. C. Gray, D. H. Pontius, Jr, and J. W. Bieber, Phys. Rev. Lett. 75, 2136 (1995).

[26] G. Zimbardo, P. Veltri, G. Basile, and S. Principato, Phys. Plasmas 2, 2653 (1995).

[27] P. Pommois, P. Veltri, and G. Zimbardo, Phys. Rev. E 59, 2244 (1999).

[28] A. Shalchi and B. Weinhorst, Adv. Space Res. 43, 1429 (2009).

[29] A. Shalchi and G. Qin, Ap\&SS 330, 279 (2010).

[30] A. Shalchi, CoPP 51, 920 (2011).

[31] A. Shalchi, Phys. Plasmas 17, 082902 (2010a).

[32] B. Guest and A. Shalchi, Phys. Plasmas 19, 032902 (2012).

[33] S. Corrsin, in Atmospheric Diffusion and Air Pollution, Advanced in Geophysics, Vol. 6, edited by F. Frenkiel and P. Sheppard (Acadenic, New York, 1959).

[34] J. W. Bieber, W. H. Matthaeus, C. W. Smith, W. Wanner, M.-B. Kallenrode, and G. Wibberenz, Astrophys. J. 420, 294 (1994).

[35] I. S. Gradshteyn and I. M. Ryzhik, Table of integrals, series, and products (Academic Press, New York, 2007).

[36] M. Abramowitz and I. A. Stegun, Handbook of Mathematical Functions (Dover, New York, 1974).

[37] W. Paulsen, Asymptotic Analysis and Perturbation Theory (CRC Press, Florida, 2014).

[38] I. Kourakis, R. C. Tautz, and A. Shalchi, J. Plasma Physics 75, 183 (2009).

[39] A. Shalchi, Astrophys. J. 720, L127 (2010b). 